\title{
Linx
}

Revue des linguistes de l'université Paris X Nanterre

64-65 | 2011

Les genres de discours vus par la grammaire

\section{Les consécutives intensives : un schéma syntaxique commun à plusieurs genres de discours}

Jean-Michel Adam

\section{OpenEdition}

\section{Journals}

Édition électronique

URL : http://journals.openedition.org/linx/1407

DOI : 10.4000/linx.1407

ISSN : 2118-9692

Éditeur

Presses universitaires de Paris Nanterre

\section{Édition imprimée}

Date de publication : 1 juillet 2011

Pagination : 115-131

ISSN : 0246-8743

\section{Référence électronique}

Jean-Michel Adam, «Les consécutives intensives : un schéma syntaxique commun à plusieurs genres de discours », Linx [En ligne], 64-65 | 2011, mis en ligne le, consulté le 19 avril 2019. URL : http:// journals.openedition.org/linx/1407; DOI : 10.4000/linx.1407 


\title{
Les consécutives intensives : un schéma syntaxique commun à plusieurs genres de discours
}

\author{
Jean-Michel Adam \\ Université de Lausanne
}

\section{Grammaire, style, genres}

Les formes de langue et les formes types d'énoncés, c'est-à-dire les genres du discours, s'introduisent dans notre expérience et dans notre conscience conjointement et sans que leur corrélation étroite soit rompue. Apprendre à parler c'est apprendre à structurer des énoncés (parce que nous parlons par énoncés et non par propositions isolées et, encore moins, bien entendu, par mots isolés). Les genres du discours organisent notre parole de la même façon que l'organisent les formes grammaticales (syntaxiques). (Bakhtine 1984 : 285)

Au lieu d'étudier les configurations de marques microlinguistiques qui caractérisent éventuellement un genre, le présent article aborde la question théorique des liens entre un schéma syntaxique, ses valeurs sémantico-pragmatiques et un ou plusieurs genres de discours dont il peut être un indicateur important. Le schéma syntaxique choisi correspond à une forme particulière de subordonnée consécutive que Muller qualifie de «consécutive quantifiée » (1996) et qu'Hybertie range dans les «systèmes corrélant intensité et consécution » (1996). Je parle de «faits grammaticostylistiques » pour signifier que je me situe dans le champ de ce que Bakhtine dit à plusieurs reprises des rapports entre grammaire et stylistique :

La grammaire et la stylistique se rejoignent et se séparent dans tout fait de langue concret qui, envisagé du point de vue de la langue, est un fait de grammaire, envisagé du point de vue de l'énoncé individuel est un fait de stylistique. Rien que la sélection 
qu'opère le locuteur d'une forme grammaticale déterminée est déjà un acte stylistique. Ces deux points de vue sur un seul et même phénomène concret de langue ne doivent cependant pas s'exclure l'un l'autre, ils doivent se combiner organiquement (avec le maintien méthodologique de leur différence) sur la base de l'unité réelle que représente le fait de langue [...]. (Bakhtine, 1984 : 272)

C'est dans des textes inscrits discursivement dans des genres que le fait microlinguistique observé fait sens et fonctionne de façon systémique. Je choisis de traiter comparativement les fonctionnements et les valeurs sémantico-pragmatiques d'un schéma syntaxique présent dans des genres littéraires et non littéraires. Ce choix de corpus me permet de me démarquer des démarches habituelles qui séparent, comme dans la tradition allemande, les genres littéraires (Gattung) et les textes utilitaires et spécialisés (Textsorten ou Textmuster). Je suis la position exprimée très clairement par Todorov dans Les genres du discours:

Chaque type de discours qualifié habituellement de littéraire a des "parents » non littéraires qui lui sont plus proches que tout autre type de discours «littéraire ». [...] Ainsi l'opposition entre littérature et non-littérature cède la place à une typologie des discours.

[...] À la place de la seule littérature apparaissent maintenant de nombreux types de discours qui méritent au même titre notre attention. Si le choix de notre objet de connaissance n'est pas dicté par de pures raisons idéologiques (qu'il faudrait alors expliciter), nous n'avons plus le droit de nous occuper des seules sous-espèces littéraires, même si notre lieu de travail s'appelle «département de littérature » (française, anglaise ou russe). (1978:25)

Le dernier état de la théorie des genres que je défends depuis une dizaine d'années est présenté dans Adam et Heidmann, 2009. Nous définissons le concept de généricité comme l'appartenance d'un texte non pas à un seul genre (cas rare), mais à plusieurs au sein d'un système de genres propre à un état donné de l'interdiscours d'une formation sociodiscursive particulière. Dès qu'il y a texte, c'est-à-dire reconnaissance du fait qu'une suite d'énoncés forme un tout de communication, il y a effet de généricité, c'est-à-dire inscription de cette suite d'énoncés dans au moins une classe de discours et ceci de façon convergente ou divergente entre la production et la réception. Je rappelle que, selon moi, un genre est une catégorie pratique-empirique régulatrice des énoncés en discours. C'est par ailleurs une catégorie prototypique, qui tolère des réalisations plus ou moins déviantes et ne fonctionne pas en soit/soit, mais en plus ou moins. Bally formulait cette exigence des modèles linguistiques dans Le langage et la vie :

Les notions sur lesquelles opère la linguistique, les classes qu'elle établit, ne sont pas des entités fixées une fois pour toutes : d'une classe à l'autre, d'une notion à la notion contraire, on passe toujours par de larges zones intermédiaires, si bien que les lois linguistiques devraient se borner à formuler des variations concomitantes, selon le schéma : plus... plus, plus... moins, dans la mesure où, etc. (Bally, 1965 : 75)

Par ailleurs, à propos des proverbes et dictons, Greimas notait déjà, dans $D u$ sens, que la recherche des caractères formels du genre et des sous-genres est difficile dans la mesure où ceux-ci «se rencontrent rarement tous dans un seul exemple» (1970 : 311) et il ajoutait fort justement, de façon plus générale : 
Cela n'étonnera pourtant pas le linguiste : l'existence de leste (qui ne réalise pas formellement l'opposition masculin vs féminin), ou de voix (où la distinction singulier vs pluriel n'est pas marquée, même graphiquement), ne remet pas en question les catégories du genre et du nombre; ni l'historien de l'art: les différentes cathédrales gothiques ne réunissent presque jamais non plus tous les traits distinctifs du gothique. $(1970: 311)$

Ce type de réflexion permet de ne plus appuyer les classifications sur la recherche de critères définitoires en termes de conditions nécessaires et suffisantes, mais sur des groupements d'attributs d'importance variable. Les catégories génériques sont constituées de faisceaux de marques et on ne mesure jamais que l'appartenance graduelle d'un texte à une catégorie. Ma position est très proche du concept allemand de Textmuster (trame ou moule textuel) en raison de son caractère dynamique, de la prise en compte qu'il implique de l'hétérogénéité constitutive de textes réalisés et de l'attention à leur production et à leur lecture-interprétation.

Dans « Consécutives intensives et mouvement du sens dans quelques contes de Perrault, Grimm et Andersen », Thérèse Jeanneret parle «du caractère typique de la configuration intensive consécutive pour le conte » en général $(2005: 20)$ et elle ajoute fort justement : «Bien évidemment, le conte n'est pas le genre exclusif d'occurrence de ce schéma. Plutôt, notre configuration intensive consécutive est un paramètre à prendre en compte parmi d'autres pour la caractérisation du genre conte » (2005: 12). Je montrerai que la fréquence élevée de ce schéma syntaxique est certes une des caractéristiques des contes en prose de Perrault (corpus 3), mais qu'elle est également présente dans un genre narratif bref du XVII siècle comme les nouvelles historiques et galantes (corpus auquel je ne ferai, ici, qu'allusion). La fréquence élevée des intensives consécutives dans le genre des insultes rituelles a été identifiée par William Labov (corpus 1) et je reviens ici sur le glissement de ce genre vers l'histoire drôle, dont j'ai parlé dans le chapitre 6 de Adam 1999. Par ailleurs, dans une étude parue en 2005, j’ai constaté la fréquence de ces mêmes formes dans le discours publicitaire (Adam, 2005b) et j’ai rapproché ce fonctionnement des hypothèses générales sur la rhétorique épidictique de la publicité explorée dans Adam et Bonhomme, 1997 (corpus 2).

\section{Les intensives consécutives dans le genre de l'insulte rituelle $<$ corpus $1>$}

Dans le chapitre 8, «Rules for Ritual Insults », du premier volume de Language in the Inner City: Studies in the Black English Vernacular (1972 : 297-351; trad. fr. 1978), Labov a observé, chez les jeunes noirs des «quartiers réservés » de New York, Boston, Detroit, Philadelphie, Washington, etc., une pratique discursive «remarquablement identique dans toutes les communautés noires tant par la forme que par le contenu des insultes et par les règles qui régissent l'interaction verbale » (1978:233). Ces vannes ou charres concernant les proches parents sont des formes d'injures dépragmatisées ou insultes rituelles, appelées sounds, dans la culture vernaculaire noire américaine (également woofing, screaming, etc.). Les deux participants qui s'engagent dans une joute verbale d'insultes rituelles se renvoient l'un à l'autre, sous forme de " coup ", une insulte que le groupe spectateur des pairs évalue. Le duel se prolonge, en dépit de la violence des 
insultes, sans qu'aucun participant ne se sente injurié. Le vainqueur de l'affrontement verbal est celui qui parvient à répliquer en utilisant plus de "vannes» que son adversaire ou de meilleures, plus fortes, plus surprenantes, plus inventives.

Dans cette étude sociodiscursive, Labov démontre qu'étudier une conduite langagière revient à identifier des contextes d'interaction sociale, des données sémantiques et microlinguistiques ainsi qu'une forme textuelle de composition. Il a aussi mis en place une procédure de description fine d'un genre de discours. Ce genre présente des caractéristiques microlinguistiques très variées, mais une forme a retenu l'attention de Labov, qui la considère même comme «typical sound» (1972: 336) : une forme simple de base (1) qui peut être elliptique (ellipses recouvrables de « is » et de « that » comme en (2); ci-après type 1) voire plus complexe (comme en (3); ci-après type 2) :

(1) Your mother is so ugly that she looks like the Abominable Snowman. (1972: 336)

(2) Your mother, so old, she fart dust. (1972: 336)

(3) Bell grandmother got so many wrinkles in her face, when they walk down the street, her mother would say, «Wrinkles and ruffles ». (1972:346)

Fort éloignée de la brutale simplicité de l'injure classique, la sophistication sémantique et grammaticale de cette forme met en évidence la compétence linguistique des sujets. Dans l'argumentation de Labov, le «typical sound» manifeste certaines propriétés du Black English Vernacular.

Cette pratique discursive a déferlé, au début des années 1990 - en étroite corrélation avec les modes vestimentaires adolescentes venues des USA - dans les cours de récréation des collèges et les lycées français. Mais le genre identifié par Labov a progressivement, en Angleterre puis en France, pris une forme proche de l'histoire drôle. Avec Ta mère, volume publié par Arthur, dans une collection de l'éditeur Michel Lafon, en 1995, - dont seront extraits les exemples français cités ci-après -, la mise en recueil ou répertoire d'histoires drôles écrites a matérialisé le déplacement du genre de sa communauté discursive d'origine vers d'autres formations socio-langagières. Si la fonction du genre a changé en passant du cadre sociodiscursif de l'insulte rituelle au genre de l'histoire drôle. Le passage au genre second s'est traduit par la sélection du schéma syntaxique défini comme prototypique par Labov.

Les variations observées dans le genre premier sont, selon Labov, dérivées d'une forme corrélative de base présentant une construction corrélative consécutive simple (type 1) ou complexe (type 2).

Type 1

Proposition $\mathrm{p}: \mathrm{X}$ être/avoir... SI/TELLEMENT [a] >> QUE $>>$ proposition $\mathrm{q}$ Proposition p comportant un intensif [a] >> corrélation de conséquence $>>$ proposition $q$ 
Labov considère (1) (2) et (4) comme des exemples de cette forme simple :

(4) Bell grandmother so-so-so ugly, her rag is showin'. (Labov 1972 : 346)

En (4) l'ellipse de « is » est un aspect de l'oralité et celle de «that » tient au fait que la corrélative intensive accentuée par la répétition de «so » n'a pas besoin de la présence en surface de la marque de connexion. Cette forme textuelle réalise la construction progressive d'une représentation discursive descriptive : une propriété [a] (attribute) est attribuée à un individu $\mathrm{X}$, cible (target) de l'acte de discours : le destinataire lui-même (pronom personnel $t u, t^{\prime}$ ) ou une partie de son corps (tes dents, tes oreilles, ta bite) ou, le plus souvent, un proche parent (ta mère, ton père, ton frère, ta scur, ta famille) ; ce que Labov schématise sous une forme $\mathrm{X}(\mathrm{B})$ où $\mathrm{B}$ est le destinataire et $\mathrm{X}$ le parent proche de B. Cette propriété [a] est fortement dépréciative et elle est, de surcroît, modalisée par un adverbe (SO en anglais, équivalent de nos SI intensif, TELLEMENT et TANT). $\mathrm{Au}$ lieu de chercher, par un emploi classifiant, à faire entrer la cible X dans des classes délimitables porteuses d'informations (classe des êtres vieux « so old », laids « so ugly », etc.), il s'agit, par ce moyen syntaxique, de la dévaloriser hyperboliquement. Par un acte d'énonciation singulier, le locuteur attribue à la cible une propriété [a] portée à un extrême degré d'intensité négative.

Du fait de la présence des adverbes SI ou TELLEMENT, une contrainte de complétude syntaxique s'exerce sur l'énoncé. Si cette contrainte de complétude des systèmes consécutifs, proclamée un peu rapidement par les grammaires, peut être sérieusement remise en cause (voir à ce propos Noailly, 1998 et plus loin), elle apparaît, dans le genre de l'insulte rituelle comme de l'histoire drôle qui en dérive, comme une contrainte absolue. Nous sommes en présence d'une structure corrélative dans laquelle une première proposition [p] est corrélée à une proposition consécutive postposée [q], introduite par QUE. Cette proposition constitue l'assertion la plus originale, la pointe rhétorique qui clôt l'intervention. Comme le dit Labov, couplée à la propriété attribuée à la cible $[\mathrm{X}(\mathrm{B})]$ par le quantifieur «SO... THAT », la proposition $\mathrm{q}$ exprime le degré auquel $\mathrm{X}(\mathrm{B})$ possède la propriété [a] :

(5) Ta mère est TELLEMENT plate QU'on pourrait la faxer.

(6) Ta mère est SI pauvre QUE c'est les éboueurs qui lui donnent des étrennes.

Un connecteur peut venir encore renforcer la proposition $\mathrm{q}$ :

(7) Ta mère est SI féroce QUE même les pitbulls changent de trottoir.

(8) Ta famille est TELLEMENT fauchée QUE chez toi les pendules veulent même pas donner l'heure.

Les enchâssements syntaxiques peuvent être plus complexes : «Une des façons d'atteindre la perfection en matière de vanne consiste à [introduire] un fort enchâssement à gauche qui suspend la proposition finale »(Labov, 1978 : 283) :

Type 2 : Prop. $\mathrm{p}: \mathrm{X}$ est TELLEMENT/SI [a]... QUE \{ QUAND [b] \} prop. $\mathrm{q}$

(3) Bell grandmother got so many wrinkles in her face, when they walk down the street, her mother would say, «Wrinkles and ruffles ». 
L'ellipse de «that » s'accompagne d'une complexification des enchâssements syntaxiques avec l'insertion de la subordonnée introduite par «When» et du segment de discours direct rapporté qui teint lieu de proposition q. Soit un retardement de l'énoncé de q par insertion d'un circonstant à valeur cadrative (hypothétique ou temporelle) [b] qui accentue la causalité déjà impliquée par la consécutive. Cette structure ternaire est la forme la plus fréquente dans le corpus d'histoires drôles sur lequel j’ai travaillé. L'ordre des propositions est déterminé par le renforcement de effet rhétorico-stylistique de "pointe ». L'élément le plus inattendu et donc le plus inventif du point de vue de la créativité verbale est retardé et placé en position rhématique :

(9) Ton père est TELLEMENT con QUE QUAND je lui ai dit : « Regardez, une mouette morte ! ", il a levé la tête en l'air et m'a demandé : « Où ça ? »

(10) Ta mère a des jambes TELLEMENT énormes QUE LE JOUR OÙ elle a retiré ses bas résille pour se baigner dans la mer, on l'a chopée pour utilisation illicite de filets dérivants.

(11) Ta bite est SI petite QUE SI je la présentais au tribunal, on la rejetterait pour manque de preuve.

La fonction sémantico-pragmatique de la structure textuelle de ces énoncés est ainsi résumée par Labov :

Les vannes sont dirigées contre une cible très proche de l'adversaire (ou contre l'adversaire lui-même), mais, par une convention sociale, on admet que les attributs qu'elles désignent n'appartiennent en réalité à personne. Pour le dire comme Goffman, le maintien d'une distance symbolique permet d'isoler l'échange des conséquences qu'il pourrait avoir. Ce statut rituel, les règles que nous avons données ainsi que les diverses échappées vers le bizarre et le farfelu ont précisément pour effet de le préserver. (1978: 287-288)

En d'autres termes, la propriété énoncée est portée à un tel seuil d'intensité qu'elle ne peut pas exister dans le monde réel et donc l'insulté ne peut ressentir l'injure comme un acte de discours mettant sérieusement sa «face » en péril. Il sait, en raison du caractère hyperbolique de l'intensive, que l'énoncé est fictionnel. L'insulte rituelle présente un glissement générique de la sphère réelle de l’insulte personnelle vers la sphère fictionnelle du jeu et de la joute rituelle. De ce fait, un double glissement intervient : le genre premier oral de l'injure-insulte est transformé en vanne ou insulte rituelle plus élaborée et, par ailleurs, le genre de l'insulte rituelle est transformé en genre second écrit dans le recueil d'histoires drôles. L'histoire drôle en forme (calque) d'insulte rituelle prolonge le mouvement fictionnel de distanciation. Le changement radical de formation discursive, de langue et de genre conserve la texture grammaticostylistique et compositionnelle observée dans le genre premier oral et ne retient même que cette forme. L'histoire drôle est elle-même une forme fictionnelle déclarée. 


\section{Les intensives consécutives dans le discours publicitaire $<$ corpus $2>$}

Le discours publicitaire recourt fréquemment à deux constructions intensives. On trouve des formes autonomes d'emploi de SI intensif absentes du corpus précédent :

(12) Nouvelle Citroën Xantia, le Break.

Très Break, et pourtant SI berline.

Elégance raffinée, espace volumineux, ligne stylée, très haut niveau de confort jusque dans les moindres détails $[\ldots]$

On observe aussi un grand nombre de corrélations de l'intensif et de la consécution qui confèrent aux adverbes intensifs TELLEMENT et SI une valeur de connecteur, selon le schéma syntaxique dont nous venons de parler:

(13) KANTERBRÄU EST SI BONNE QU'ON NE PEUT S'EN PASSER

(14) La Voiture d'Ulysse. (ou comment la Chrysler Simca 1307/1308 démontre brillamment son goût pour les longs voyages.)

[...] Ses sièges étaient SI confortables et sa marche TELLEMENT silencieuse QUE jamais la fatigue ne venait clore les yeux de ses passagers.

(15a) SI économique pour la

VAISSELLE

PAIC

CITRON

DEGRAISSE

PLUS VITE

(énoncé présent sur l'étiquette du produit)

$\left(15^{b}\right)$ Paic Citron dégraisse SI bien QU'aussitôt la vaisselle étincelle et quelques gouttes suffisent.

(slogan de pied, base line)

(16a) LES FEUILLETINES AOSTE

SI finement tranchées QU'on les savoure du bout des doigts. (en accroche)

(16 $\left.{ }^{\mathrm{b}}\right)$ Quand on saisit du bout des doigts une Feuilletine Aoste, SI fine ET SI délicatement chiffonnée, quand on savoure son goût raffiné et incomparable... on ne peut pas s'empêcher d'en déguster une autre, puis encore une autre !

(16c) On peut ainsi en profiter pour partir à la découverte de leurs différentes saveurs : jambon cru, jambon cru fumé ou saucisson. Le secret des Feuilletines Aoste? Elles sont SI finement tranchées QUE leurs saveurs les plus subtiles peuvent pleinement s'épanouir. C’est vraiment difficile de résister aux Feuilletines Aoste...

Les constructions sont très variées : expression ou ellipse du verbe " être », corrélation en SI... QUE... ou absence de continuation. On retiendra de ces emplois 
attestés que SI intensif présente souvent un redoublement soit coordonné, soit simplement juxtaposé de l'adverbe et de l'adjectif. Les exemples (12), ainsi que (15a) et $\left(16^{\mathrm{b}}\right)$, présentent un emploi intensif autonome de SI que l'on peut dire exclamatif, même si la marque de l'exclamation n'est presque jamais présente. Les autres comportent tous une corrélation consécutive de type SI (TELLEMENT)... QUE. Dans les propositions en SI autonome (sortes de corrélatives tronquées), l'intensif et l'exclamation l'emportent sur le liage logico-grammatical. En quelque sorte, cette syntaxe expressive découle de l'expression d'une intensité telle que rien ne peut et n'a besoin d'être prédiqué à la suite (ellipse de q).

Les énoncés qui suivent le nom du produit, en $\left(15^{\mathrm{a}}\right)$ et $\left(15^{\mathrm{b}}\right)$, présentent deux structures phrastiques parallèles. Une structure comparative elliptique : «Paic Citron dégraisse PLUS VITE» $\left(15^{a}\right)$ - soit une ellipse de QUE les produits $\mathrm{Y}, \mathrm{Z}-$ et une structure corrélative intensive: "Paic Citron dégraisse SI BIEN QU'aussitôt la vaisselle...» (15b). En (15a), la comparaison («plus») se transforme, du fait de l'absence de comparé, en intensif. Ce procédé correspond à une procédure publicitaire parfaitement commentée, dès la fin des années 1940, par Leo Spitzer :

La prédilection que montre la publicité pour le superlatif reflète ce monde d'optimisme et d'idéalisme qu'elle déroule à nos yeux ; chacun des produits vantés est supposé être le meilleur de sa sorte, depuis le pain le plus savoureux des ÉtatsUnis jusqu'à la voiture la plus parfaite au plus bas prix. Ce règne sans partage du superlatif, que ne met en question aucune comparaison factuelle (puisque la loi interdit de dénigrer les produits de ses concurrents), tend à abolir toute différence entre forme superlative et forme emphatique : «le meilleur» devient égal à « un très bon » $[\ldots] .(1978: 163)$

La première partie de $\left(15^{\mathrm{a}}\right)$ est une sorte de construction détachée (CD) qui correspond à un énoncé de type : «Paic Citron (EST) SI économique pour la vaisselle». En remplaçant SI par TRES, ce segment intensif serait clairement une construction détachée : «TRĖS économique pour la vaisselle (CD), Paic Citron dégraisse PLUS vite 》. Avec SI, il est plus difficile de parler d'une CD. De toute évidence, l'intensif SI autonomise beaucoup plus que TRES le segment qu'il modalise. Ce qui n'est plus le cas, bien sûr, quand SI est corrélé à QUE, comme dans $\left(15^{\mathrm{b}}\right)$ : «Paic Citron dégraisse SI bien QU'aussitôt la vaisselle étincelle». Soulignons, par ailleurs, que l'adjonction de « et quelques gouttes suffisent» correspond à une structure argumentative longuement commentée dans Adam et Bonhomme, 1997 : 144-148) : ce qu'on peut appeler le topos argumentatif du moins de produit (" quelques gouttes ») et le moins cher (" économique ») pour le plus d'effet («si bien »).

Comme le montre, à la suite de Spitzer, notre livre sur L'argumentation publicitaire, la publicité a quelque chose à voir non seulement avec le genre délibératif pour ce qui concerne la décision d'achat du produit, mais avec le genre épidictique des louanges : le schéma syntaxique qui sert de blâme dans le corpus d'insultes rituelles et d'histoires drôles devient moyen de l'éloge dans le corpus publicitaire. Telle est du moins la généricité rhétorique qui nous paraît caractériser le discours publicitaire : l'hyperbole positive trouve son expression linguistique la plus accomplie dans l'intensif en SI / TELLEMENT / TANT (QUE). En effet, de cette façon, la propriété attribuée à l'objet de valeur publicitaire est portée à son plus haut terme d'intensité, atteignant un 
seuil plus qualitatif que quantitatif. Le seuil qualitatif atteint est tellement extrême qu'il flirte hyperboliquement avec la fiction. Ce qui est dit du produit est si extraordinaire que le discours publicitaire évoque un monde sublimé. Spitzer parle d'un "paradislangage ». Les modalités d'interprétation de l'argumentation publicitaire sont comparables au mode de croyance de la superstition et de la lecture des énoncés fictionnels : "Je sais bien que c'est hyperbolique et donc faux, mais c'est quand même tellement agréable!", donc vraisemblable selon l'ordre du désir. En d'autres termes, l'interprétation de la publicité est très proche de la clausule des conteurs majorquins citée par Roman Jakobson (1963 : 239) : "Aixo era y no era ». Cela était et n'était pas est transposé en (Je sais bien que) cela est et n'est pas vrai. Ce qui nous amène au troisième corpus, celui des contes.

\section{Les hyperboliques intensives dans les contes de Perrault $<$ corpus $3>$}

L’idée d'une forme qui serait «typique» du conte doit être relativisée. D'un point de vue quantitatif, les corrélatives intensives et les intensives hyperboliques (sans corrélation syntaxique) ne se distribuent pas uniformément dans les huit contes en prose de Perrault (je prends pour base de calcul les pages du fac-similé de l'édition Barbin de 1697) :

\section{Distribution des corrélatives intensives et des intensives hyperboliques}

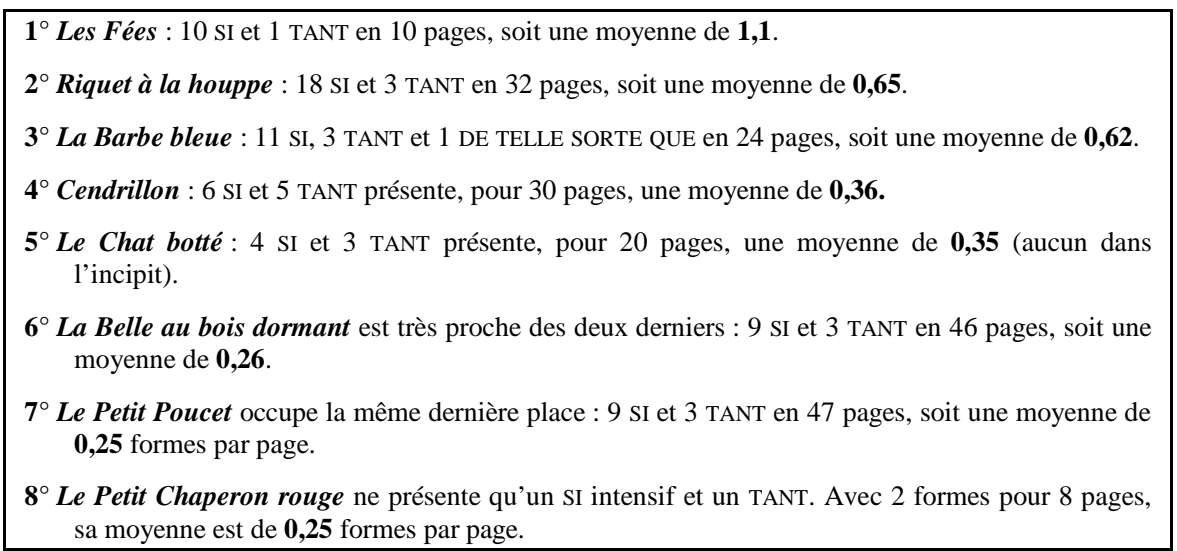

Le très court conte des Fées se détache nettement des autres. Dans son incipit (17), le milieu du récit (18) et sa fin - donnée sous ses versions de la première édition de 1697 (19) et du manuscrit d'apparat de 1695 (20) -, on observe une multiplication du même schéma syntaxique.

(17) IL ESTOIT UNE FOIS UNE veuve QUI avoit deux filles, l'aînée luy ressembloit SI FORT \& d'humeur \& de visage, QUE qui la voyoit voyoit la mere. Elles étoient toutes deux SI DESAGREABLES \& SI ORGUEILLEUSES QU'on ne pouvoit vivre avec elles. La cadette qui estoit le vray portrait de son Pere pour 
la douceur \& pour l'honnesteté, estoit avec cela UNE DES PLUS BELLES FILLES QU'ON EUST SÇEU VOIR.

(18) La bonne femme ayant bû, luy dit, vous estes SI BELLE, SI BONNE \& SI HONNESTE, QUE je ne puis m'empêcher de vous faire un don [...].

(19) Pour sa sœur elle se fit TANT haï, QUE sa propre mere la chassa de chez elle ; \& la malheureuse aprés avoir bien couru sans trouver personne qui voulut la recevoir, alla mourir au coin d'un bois. (1697)

(20) Pour sa sœur l'incivile elle se fit TELLEMENT haïr et regarder avec horreur a cause des vilaines Bestes qui luy sortoient de la bouche toutes les fois qu'elle parloit QUE sa propre mere ne pouvoit la souffrir et la chassa honteusement la malheureuse courut long temps de tous costez sans que personne voulust la recevoir et on dit qu'elle alla mourir malheureusement au coin d'un buisson. (1695)

Dans ces configurations syntaxiques, la proposition $\mathrm{p}$ comporte trois sortes d'adverbes intensifs : SI, TANT, TELLEMENT. La seconde proposition est introduit par le subordonnant marqueur de consécution QUE q. Dans les exemples (17) et (18), l'adverbe SI modifie intensivement un adjectif ou un adverbe (fort, belle, bonne, orgueilleuse, etc.) et, dans les exemples (19) et (20), les adverbes TANT et TELLEMENT opèrent la même modification intensive de verbes à l'infinitif. Notons que devant un substantif, on trouve la construction UN(E) TEL(LE), avec simple intensification d'une propriété dans Les Fées (21) et corrélation complète dans Riquet à la houppe (22) :

(21) Le fils du Roi en devint amoureux, \& considerant qu'UN TEL DON valoit mieux que tout ce qu'on pouvoit donner en mariage à un autre, l'emmena au Palais du Roi son pere, où il l'épousa.

(22) elle commença dés ce moment une conversation galante, \& soutenuë avec Riquet à la houppe, où elle brilla D'UNE TELLE FORCE, QUE Riquet à la houppe crut luy avoir donné plus d'esprit qu'il ne s'en estoit reservé pour luymême.

D’un point de vue sémantique, la conséquence exprimée dans la proposition consécutive q est sous la dépendance du seuil d'intensité exprimé par l'adverbe présent dans la proposition $\mathrm{p}$. L'adverbe intensif de la proposition $\mathrm{p}$ exprime un degré d'intensité à partir duquel la conséquence q ne peut que se produire. Ces seuils positifs ou négatifs sont toujours extrêmes et confèrent aux personnages des propriétés hors du commun.

Cette façon de porter les propriétés des personnages au-delà de ce qui peut être décrit, perçu ou pensé, préside à la mise en place du monde fictionnel des contes dès l'incipit des premier (La Belle au bois dormant), troisième (La Barbe bleüe), cinquième (Les Fées) et septième (Riquet à la Houppe) contes. La construction progressive du monde du texte passe par une structure syntaxique assez complexe. Après le prédicat d'existence (a), apparaissent une relative appositive (prédicative) ou une simple apposition qui assurent la deuxième prédication (b) et introduisent la tension narrative. La corrélation entre l'intensif (SI) et la consécutive (QUE q) est intégrée dans la relative prédicative : 
(23) (a) Il estoit une fois UN Roi \& UNE Reine

(b) QUI étaient SI faschez [...] SI faschez

(c) QU'ON ne sçauroit dire

(24) (a) Il estoit une fois UN homme

(b) QUI avait de belles maisons [...]

(c) mais par malheur cet homme avait la barbe bleue :

(d) cela le rendoit SI laid \& SI terrible

(e) QU'il n'estoit ni femme...

(17) (a) Il estoit une fois UNE veuve

(b) QUI avoit deux filles,

(c) l'aînée luy ressembloit SI fort \& d'humeur et de visage

(d) QUE qui la voyoit voyoit la mere.

(e) Elles étoient toutes deux SI desagreables \& SI orgueilleuses

(f) QU'ON ne pouvoit vivre avec...

(25) (a) Il estoit une fois UNE Reine

(b) QUI accoucha d'un fils SI laid \& SI mal fait

(c) QU'ON douta long-tems s'il...

La structure syntaxique de double intensive consécutive de (23) (SIp ${ }^{1}$ \& $\mathrm{SIp}^{2}$ QUE q) se retrouve dans (24), (17) et (25). Dans Le Petit Poucet, en l'absence de corrélative intensive, une relative (b) est associée dans la phrase suivante à une comparaison intensive (c) :

(26) (a) IL ESTOIT UNE FOIS UN Bucheron \& UNE Bucheronne, (b) QUI avoient sept enfans tous Garçons. L'aîné n'avoit que dix ans, \& le plus jeune n'en avoit que sept. (c) On s'estonnera que le Bucheron ait eu TANT D'ENFANS EN SI PEU DE TEMPS, mais c'est que sa femme alloit vite en besongne, \& n'en faisoit pas moins de deux à la fois.

Ces effets de sens transparaissent à l'examen de la récriture de l'incipit des Fées. Le début, dont (17) donne la récriture de 1697, se présentait ainsi en 1695 :

(27) Il estoit une fois un gentil homme qui étant veuf d une femme TRÈS DOUCE ET TRÈS HONNESTE et ayant eu d'elle une fille toute semblable a sa mere epousa en secondes nopces une femme TRÈS HAUTAINE ET TRÈS FACHEUSE qui avoit une fille de sa meme humeur AUSSI laide ET AUSSI maussade QUE l'autre étoit belle et civile.

En remplaçant l'adverbe TRÈS ( très hautaine \& très fâcheuse ») par une corrélative intensive, Perrault accentue, en 1697, la causalité («SI désagréable \& SI orgueilleuse QUE ...»). L'adverbe intensif TRÈS, dans l'incipit de 1695, ne déclenche syntaxiquement pas de corrélation consécutive, alors que la présence d'un SI intensif ouvre la possibilité d'une conséquence narrative.

Avec les adverbes intensifs BIEN (plus de 70 occurrences), FORT (44 occurrences), TRÈS (7 occurrences), l'accroissement est simplement quantitatif. Avec SI (66 occurrences) et TANT (une vingtaine d'occurrences), il ne s'agit plus de la même échelle d'intensité. TRÈS, comme ASSEZ ou TROP, «manifeste une variation de degré 
sur une échelle d'intensité objective, à laquelle est rapporté l'adjectif ou l'adverbe auquel il est incident» (Plantin, 1985 : 42). SI, en revanche, ne situe plus l'intensité sur une telle échelle; il signale qu'un seuil a été atteint et le dépassement de ce seuil a quelque chose à voir avec le monde de la fiction merveilleuse. C'est en raison de cette valeur qualitative hyperbolique que SI et TANT s'adaptent aussi bien au monde du conte merveilleux. Ce sont, à la fois, des marqueurs de fictionnalité qui affectent sa sémantique et de causalité narrative qui densifient sa grammaire narrative.

Cette accentuation de la causalité narrative est flagrante dans La Barbe bleue:

(28) Elle fut SI PRESSÉE de sa curiosité [p], QUE sans considerer qu'il estoit malhonneste de quitter sa compagnie, elle y descendit par un petit escalier dérobé [q], \& AVEC TANT DE PRÉCIPITATION [p], QU'elle pensa se rompre le cou deux ou trois fois [q].

(29) Les voisines \& les bonnes amies n'attendirent pas qu'on les envoyast querir pour aller chez la jeune Mariée [q], TANT ELLES AVOIENT D’IMPATIENCE de voir toutes les richesses de sa Maison [p] [...].

(30) [...] elle n’en pouvoit venir à bout [q], TANT ELLE ESTOIT ÉMEUË [p]. (6869)

Dans ces deux derniers exemples, l'intensif TANT est employé sans marqueur de corrélation. Cette ellipse de QUE s'explique par l'inversion des propositions : la conséquence [q] précède la cause marquée par l'intensif $[\mathrm{p}]$ et le marqueur TANT assume donc à lui seul la corrélation consécutive.

Dans la conséquence ajoutée à (17) en 1697 («SI désagréables \& SI orgueilleuses QU'on ne pouvait vivre avec elles»), les choix de la négation du verbe modal ("ne pouvait ») et l'emploi du pronom indéfini ON posent l'impossibilité non seulement comme hyperbolique, mais comme collectivement admise. En se combinant aux intensifs SI et TANT, ce caractère en quelque sorte endoxal des valeurs fait du monde du conte un univers dans lequel il n'y a pas de place pour l'incertitude : l'élévation extrême de la cadette et la déchéance tout aussi extrême de l'aînée apparaissent comme légitimes selon un ordre hyperbolique des choses. Cette interprétation confirme la description polyphonique de Plantin, qui parle d'un «intensifieur discursif » :

S'appuyant sur une intensité "pré-énonciative», au sens ou le degré d'intensité (éventuellement élevé) n'est pas attribué à l'adjectif ou à l'adverbe du fait de SI : cette intensité est rapportée, citée par SI, l'attribution étant le fait d'un acte de discours antérieur à l'énoncé en SI. SI marque la pluralité des voix dans le discours (1985 : 42).

Dans cette analyse, les énoncés comportant un «intensifieur discursif» évoquent une représentation déjà disponible en mémoire. À la suite de Dominicy, Plantin rapproche ce mécanisme discursif de l'amplification rhétorique et de l'accord sur les valeurs qui caractérisent le discours d'éloge et de blâme épidictique. Appliquée à notre corpus, cette forme épidictique d'évocation d'une propriété correspond aux personnages hyperboliquement «beaux» ou «laids», «bien» ou "mal» éduqués, «bons » ou «méchants », « louables » ou « blâmables » qui peuplent les contes.

La construction peut être intensive, sans marque syntaxique de la corrélation, comme dans (31) et dans la réplique immédiate de la jeune fille des Fées (32) : 
(31) $[\ldots]$ sa mere la gronda de revenir SI tard de la fontaine.

(32) Je vous demande pardon, ma mere, dit cette pauvre fille, d'avoir tardé SI longtemps $[\ldots]$.

Dans les deux cas, la conséquence exprimée avant la cause (se faire gronder, demander pardon) a trait à l'acceptation par les personnages impliqués de la cause (avoir tardé à revenir à la maison) et du système de valeurs sous-jacent: on doit s'excuser d'un retour tardif et il est normal de se faire reprocher son retard par sa mère. Ce phénomène est particulièrement sensible dans le cas (rare) où le connecteur PUISQUE assure la corrélation :

(33) Vous n'estes guere honneste, reprit la Fee, sans se mettre en colere : \& bien, PUISQUE vous estes SI PEU obligeante [p], je vous donne pour don, qu'à chaque parole que vous direz, il vous sortira de la bouche ou un serpent ou un crapau [q].

Marqueur d'un raisonnement plus que d'une simple causalité, le connecteur diaphonique PUISQUE (Adam, 1990 : 243-246) présente la proposition p comme une raison déjà admise par l'interlocuteur de conclure par la proposition q. Et, dans p, la présence du SI intensif s'associe parfaitement au caractère admis de la proposition induite par PUISQUE.

La corrélation peut aussi être prise en charge par une construction détachée en -ANT, génératrice de causalité et qui se combine au SI intensif. Ainsi à la fin des Fées:

(34) Le fils du Roi qui revenoit de la chasse, la rencontra, \& [p] la voyant SI belle, [q] luy demanda ce qu'elle faisoit là toute seule \& ce qu'elle avoit à pleurer.

(35) Le fils du Roi en devint amoureux, \& [p] considerant qu'UN TEL don valoit mieux que tout ce qu'on pouvoit donner en mariage à un autre, [q] l'emmena au Palais du Roi son pere, où il l'épousa.

Dans ce type de construction détachée (soulignées ci-dessus), p devient cause de q: c'est PARCE QUE $\mathrm{p}$ (propriété marquée par l'intensif), QUE q: le fils du roi s'intéresse à la roturière rencontrée dans la forêt et l'emmène bien vite au palais du roi son père pour l'épouser.

Ce fonctionnement de SI intensif a été identifié par Spitzer, qui repère, chez Racine, un SI et un TANT qu'il appelle «d'affirmation forte» et dont il dit qu'ils permettent de "prendre l'interlocuteur à témoin », de "[faire] appel à des témoins et à un jugement étranger» $(1970: 219)$. Soulignant l'analogie avec la formule toute faite des correspondances («votre si charmante lettre »), Spitzer insiste sur le fait que les SI intensifs «présupposent la familiarité du lecteur avec la situation» (1970: 221). Dans cette analyse, il retrouve la même idée de complicité et de postulation d'un savoir partagé par le narrateur et ses lecteurs que celle que dégage Plantin :

Les énoncés en SI [...] sont polyphoniques, dans la mesure où la voix qu'ils font entendre n'est pas celle du locuteur mais celle de la communauté, du « ON » qui se matérialise par la voix concrète du locuteur. (1985:43).

Lorsque le narrateur des contes de Perrault écrit que le fils du meunier du Chat botté «ne pouvoit se consoler d'avoir un si pauvre lot» ou que la reine de Riquet à la 
houppe était « bien affligée d'avoir mis au monde un si vilain marmot», le déterminant UN s'associe à SI pour former une sorte de reformulation générique ou prototypique du summum négatif d'un bien misérable héritage ou d'une extrême laideur. D’une manière comparable, chaque fois que, sans corrélation, SI est antéposé à un adjectif comme belle ou pawvre, le narrateur nous invite à admirer ou à déplorer avec lui le caractère exceptionnellement positif ou négatif de la situation des personnages. Ces formes linguistiques demandent en quelque sorte aux lecteurs «de cautionner la légitimité de la qualification intensive, et par conséquent l'échelle de valeur impliquée par la narration » (Seylaz, 1980 : 49). Ainsi le lecteur est supposé partager la même idée de la beauté, de la laideur ou de la pauvreté que le narrateur et ses personnages. Perrault fait reposer la logique du récit sur une communauté de valeurs supposées admises et transparentes, ce qui nous place au cœur de la fonction sémantique du schéma syntaxique des intensives simplement hyperboliques et des corrélatives intensives.

Le Petit Chaperon rouge n'est pas par hasard le texte qui présente le moins de corrélatives intensives de tout le corpus. Cela tient à son étrangeté et à son dénouement aussi effroyable qu'injuste pour la petite fille. La causalité narrative troublée de ce récit faussement simple déplace les effets de sens sur un autre plan.

Les intensifs et les consécutives intensives ont pour double fonction, d'une part, de clarifier la causalité narrative en en renforçant la logique et, d'autre part, de caractériser le monde du conte comme un monde hyperbolique, de ce fait distinct de la vie ordinaire. Ce dernier point, qui fait partie des conclusions de l'analyse de Thérèse Jeanneret (2005: 20-21), doit toutefois être nuancé. En travaillant sur des nouvelles historiques galantes, j’ai constaté la présence des mêmes formes intensives que celles que j’avais observées dans le corpus contes-de-Perrault. Je n'ai pas la place de démontrer l'hypothèse sur laquelle je travaille actuellement: le schéma syntaxique observé et les formes d'intensives hyperboliques ont probablement leur origine dans un trait caractéristique d'un sociolecte : le superlatif précieux.

Ceci nous ramène aux observations de Spitzer sur les rapports entre publicité et poésie baroque et précieuse (Adam et Bonhomme, 1097: 91-95). La présence du superlatif précieux aussi bien dans les Nowvelles Françoises de Charles Sorel que dans La Princesse de Montpensier de Madame de Lafayette et même chez Racine (où Spitzer l'a remarqué), nous interdit d'affirmer que les consécutives intensives et les formes de l'«affirmation forte» sont des caractéristiques génériques des contes. Elles le sont devenues par calque stylistique, mais, à la fin du XVII siècle, elles sont, de toute évidence, propres à diverses formes de narration passionnelle et romanesque. Les deux contes de Perrault qui portent le plus explicitement sur l'art de la parole galante (Riquet la houppe) et sur la civilité (Les Fées) ne sont pas par hasard placés très haut dans le classement établi plus haut. 


\section{Conclusion provisoire}

De cette analyse se dégagent plusieurs observations méthodologiques. Associer un genre et un schéma syntaxique ne semble pas une bonne méthode. Rien ne permet de dire que le schéma syntaxique étudié par Thérèse Jeanneret et appliqué aux contes en général soit une marque du genre. En revanche, il apparaît comme une marque syntactico-sémantique qui traverse notre corpus. La même opération sémanticopragmatique de fictionnalisation est commune aux insultes rituelles, aux histoires drôles, au discours publicitaire, aux contes et aux nouvelles historiques et galantes. La présence du schéma syntactico-sémantique de l'hyperbole fictionnalisante sert donc bien à caractériser une composante de la généricité de pratiques discursives aussi différentes par ailleurs. La reconnaissance de ce schéma syntactico-sémantique commun permet de percevoir des composantes traversantes de genres discursifs en apparence fort éloignés et qu'on ne rapprocherait pas spontanément. La présence du schéma syntaxique met sur la piste de ressemblances pragmatiques et sémantiques dans le traitement des énoncés comme fictionnels. C'est graduellement que la même forme caractérise les deux genres observés dans les corpus 1 (insulte rituelle et histoire drôle) et 3 (conte de Perrault et nouvelle historique et galante). C'est comme composante d'un ensemble systémique de marques qui restent à observer que le même schéma syntactico-sémantique peut traverser des genres aussi différents. 


\section{BIBLIOGRAPHIE}

\section{Corpus}

Perrault, Charles, 1695, Contes de ma mère Loye, fac-similé du manuscrit d'apparat, in Perrault's Tales of Mother Goose, J. Barchilon éd., New York, The Pierpont Morgan Library, 1956.

— 1697, Histoires ou contes du temps passé. Avec des moralités, fac-similé du second tirage de l'édition Barbin, Paris, Firmin Didot, 1929 ; également Slatkine Reprints, Genève, 1980.

ARTHUR, 1995, Ta mère, Paris, Michel Lafon.

\section{Références bibliographiques}

ADAM, J.-M., 1990, Eléments de linguistique textuelle, Bruxelles, Mardaga.

- 1997, "Genres, textes, discours : pour une reconception linguistique du concept de genre », Revue Belge de Pbilologie et d'Histoire $\mathrm{n}^{\circ} 75$, vol. 3, Madeleine Frédéric et Jean-Pierre Van Noppen (éds.), Bruxelles, p. 665-681.

— 1999, "Ta mère: de l'insulte à l'histoire drôle », chapitre 6 de Linguistique textuelle. Des genres de discours aux textes, Paris, A. Colin, p. 157-173.

— 2001a, «En finir avec les types de textes», in Quelles grammaires enseigner à l'école? Discours, genres, texte, phrase, Claudine Garcia, Jean-Paul Confais et Michel Grandaty (éds.), Delagrave \& CRDP Midi-Pyrénées, Paris-Toulouse, p. 25-43.

— 2001b, «Analyse des discours. Types \& genres », in Communication \& interprétation, Michel Ballabriga éd., Éditions Universitaires du Sud, coll. Champs du signe, Toulouse, p. 4259.

— 2001c, «Types de textes ou genres de discours ? Comment classer les textes qui disent de et comment faire? ", Langages $\mathrm{n}^{\circ} 141$, p. 10-27.

— 2005a, "Conte écrit et représentation du discours autre. Le cas Perrault », in Dans la jungle des discours. Genres de discours et discours rapporté, J. M. Lopez Munoz, S. Marnette \& L. Rosier éds., Cadiz, P.U.Cadiz, p. 27-44.

- 2005, : "Variété des usages de SI dans l'argumentation publicitaire », in Argumentation et communication dans les médias, Marcel Burger \& Guylaine Martel éds., Québec, Nota bene, 2005, p. 81-109.

Adam, J.M. et Bonhomme, M. 1997, L'argumentation publicitaire. Rhétorique de l'éloge et de la persuasion, Paris, Nathan, coll. «FAC».

Adam, J.M. et Heidmann, U. 2004, « Des genres à la généricité. L’exemple des contes (Perrault et Grimm)», Langages 153, p. 62-72.

— 2006, "Six propositions pour l'étude de la généricité », La Licorne n 79, coordonné par Raphaël Baroni et Marielle Macé, P.U. Rennes, p. 21-34.

— 2009, Le Texte littéraire. Pour une approche interdisciplinaire, Louvain-la-Neuve, Academia Bruylant. 
BAKHTine, M.M., 1984 [1952-1953], «Les genres du discours [langage] » \& « Le problème du texte », in Esthétique de la création verbale, Paris, Gallimard, p. 263-308 \& 309-338.

BALly, Ch. 1965 [1952], Le langage et la vie, Genève, Droz.

Greimas, A.J., 1970, Du sens, Paris, Seuil.

HyBERTIE, Ch., 1996, La conséquence en français, Gap, Ophrys.

JAkOBSOn, R., 1963, Essais de linguistique générale, Paris, Éditions de Minuit.

JEANNERET, Th., 2005, «Consécutives intensives et mouvement du sens dans quelques contes de Perrault, Grimm et Andersen », Le Français moderne, n¹-73ème année, p. 6-22.

LABOV, William 1972 : «Rules for Ritual Insults », in Language in the Inner City : Studies in the Black English Vernacular, Philadelphia, University of Pennsylvania Press, p. 297-351.

— 1978 : «Les insultes rituelles », in Le parler ordinaire, vol. 1, Paris, Minuit, p. 223-288. Muller, Cl., 1996, La subordination en français, Paris, A. Colin.

NoAilly, M., 1998, "Cet homme si aimable: à propos du si d'intensité », in Du percevoir au dire, D. Leeman et A. Boone (éds.), Paris, L'Harmattan, p. 235-244.

Plantin, Ch., 1985, «La genèse discursive de l'intensité : le cas du SI “intensif” », Langages 80, 35-53.

SEYLAZ, J.L, 1980, «Un aspect de la narration stendhalienne : la qualification intensive dans le début de Lucien Leuven », Études de Lettres n³-1980, p. 31-49.

SpITZER, L., 1970, «L'effet de sourdine dans le style classique : Racine », in Études de style, Paris, Gallimard, p. 208-335.

— 1978 [1949], « La publicité américaine comme art populaire », Poétique, n 34, p. 152-171.

TODOROv, Tz., 1978, Les genres du discours, Paris, Seuil. « L'origine des genres » est repris dans La notion de littérature, Paris, Seuil, collection « Points », 1987.

— 1981, Mikhail Bakhtine : le principe dialogique. Écrits du Cercle de Bakhtine, Paris, Seuil. 